\title{
CONTROLLABILITY AND OBSERVABILITY OF LINEAR IMPULSIVE ADJOINT DYNAMIC SYSTEM ON TIME SCALE
}

\author{
NUSRAT YASMIN, SAFIA MIRZA, AWAIS YOUNUS AND ASIF MANSOOR
}

\begin{abstract}
This paper deals with the controllability, observability of the solution of timevarying system on time scales. We obtain new results about controllability and observability and generalize to a time scale some known properties about stability from the continuous case.
\end{abstract}

\section{Introduction}

The study of controllability and observability plays an important role in the control theory. In recent years, some research dealing with study of controllability and observability for impulsive systems[10, 14, 19, 20, 24, 25]. From the control point of view, hybrid impulsive control based on the theory of impulsive dynamic systems, is an effective method in the sense that it allows stabilization of a complex system behaviors may follows unpredictable patterns Interest in impulsive control systems has grown in recent years due to its theoretical and practical significance $[4,7,15,16,26]$, but to our knowledge there are very few reports dealing with hybrid impulsive dynamical systems and the corresponding contriol problem.

We are interested with dynamic systems on time scale because this area is new progressive component of applied analysis. The theory of time scales was introduced by Stefen Hilger in his Ph.D work [11] in order to unify continuous and discrete analysis. The main idea of the study is to prove the results for dynamic equations where the domain of the unknown function is a so-called time scale, which is an arbitrary non-empty closed subset of the real numbers. By choosing the time scale to be set of real numbers, the general results yields the results concerning ordinary differential equations, on the other hand, by choosing the time scale to be set of integer, the same general results yields the results for difference equations. The study of dynamic equations on time scale has recently received a lot of attention [1, 2, 3, 20, 22, 23]. It is a useful tool to explain the differences and similarities between difference and differential equations. The theory of time scale provides a deep understanding of physical applications

2010 Mathematics Subject Classification. 34N05, 34D05, 39A12.

Key words and phrases. Time scale, controllability, observability, Gramain Matrix.

Corresponding author: Nusrat Yasmin. 
and leads to the invention of some results which are known even for the special cases of time scale. Many research papers appeared in the theory and its applications to dynamic systems; see for examples the interesting monographs of Bohner and Peterson [5, 6].Many publications to investigate the stability criteria for the solution of dynamic systems on time scales can be found in [8, 12, 21].B. Kaymakakcalan [13] established a Levinson type theorem and a Yakubovich type result on asymptotic equivalence of linear dynamic equations respectively.A. Slavik [23] showed that dynamic equation on time scales can be treated in the framework of generalized ordinary differential equations as introduced by J.Kurzweil. In recent years, there has been much research activity concerning the oscillation and nonoscillation of solution of various dynamic equations.Some authors studied impulsive dynamic systems on time scales, but only few authors have studied linear impulsive dynamic systems on time scales [9, 17].V. Lupplescuet. al. [18] studied some aspects of the qualitative theory of linear impulsive dynamic systems on time scales.They also studied the controllability and observability for a class of linear time-varying impulsive control systems on time scales [19, 20].

The purpose of this project is to lay down the foundation of linear impulsive control systems on time scales. Here we examine controllability and observability in the both, timevariant and time invariant cases. It should be noted that there have been other excellent attempts to do so $[18,19,24]$. They all examine the following linear impulsive system

$$
\left\{\begin{array}{l}
x^{\Delta}(t)=A(t) x(t)+B(t) u(t), t \in\left[t_{k-1}, t_{k}\right)_{\mathbb{T}}, \\
x\left(t_{k}^{+}\right)=\left(1+c_{k}\right) x\left(t_{k}\right), t=t_{k}, k=1,2, \ldots, \\
y(t)=C(t) x(t)+D(t) u(t), \\
x\left(t_{0}\right)=x_{0}
\end{array}\right.
$$

in an effort to generalize controllability and observability for dynamic equations. At first, this seems to be a very natural extension from the continuous and discrete cases. However, when studying controllability of the linear system (1) in a way following the corresponding proofs for the continuous and discrete systems, one must assume that the graininess function is differentiable, an assumption that is not satisfied in general for all time scales. To stepside this issue, we have altered the linear system so that it appears as

$$
\left\{\begin{array}{l}
x^{\Delta}(t)=-A_{k}^{T}(t) x^{\sigma}(t)+B_{k}(t) u(t), t \in\left[t_{k-1}, t_{k}\right) \mathbb{T}, \\
x\left(t_{k}^{+}\right)=\left(1+c_{k}\right) x\left(t_{k}\right), t=t_{k}, k=1,2, \ldots, \\
y(t)=C_{k}(t) x^{\sigma}(t)+D_{k}(t) u(t), \\
x\left(t_{0}\right)=x_{0} .
\end{array}\right.
$$

The study of controllability of the linear system (2) turns out to be feasible using the classical techniques without assuming differentiability of the graininess function. However, when 
examining observability of the linear system (2) using classical methods, one must again assume differentiability of the graininess function. But the observability study of the linear system (1) does not feature this problem.

The main purpose of this paper is to derive necessary and sufficient criteria for controllability and observability of class linear impulsive adjoint system on time scale. One of the main application of these results will be in population dynamics. Many hybrid models of different populations can be studies and check there relationship by using these criteria.

\section{Basic notion}

Let $\mathbb{R}^{n}$ be the space of $n$ dimensional column vectors $x=\operatorname{col}\left(x_{1}, x_{2}, \ldots, x_{n}\right)$ with a norm $\|\cdot\|$. Also, by the same symbol $\|\cdot\|$. We will denote the corresponding matrix norm in the space $M_{n}(\mathbb{R})$ of $n \times n$ matrices. If $A \in M_{n}(\mathbb{R})$, then we denote by $A^{T}$ its conjugate transpose. We recall that $\|\cdot\|:=\sup \{\|A x\| ;\|x\| \leq 1\}$ and the following inequality $\|A x\| \leq\|A\|\|x\|$ holds for all $A \in M_{n}(\mathbb{R})$ and $x \in \mathbb{R}^{n}$.

By a time scale $\mathbb{T}$ we mean any closed subsets of $\mathbb{R}$. Since a time scale $\mathbb{T}$ is not connected in generally, we need the concept of jump operators. The forward jump operator $\sigma: \mathbb{T} \rightarrow \mathbb{T}$ is defined by $\sigma(t):=\inf \{s \in \mathbb{T}: s>t\}$, while the backward jump operator $\rho: \mathbb{T} \rightarrow \mathbb{T}$ is defined by $\rho(t)=\sup \{s \in \mathbb{T}: s<t\}$. In these definitions we put inf $\phi=\sup \mathbb{T}$ and $\sup \phi=\inf \mathbb{T}$. If $\sigma(t)>t$, we say $t$ is a right-scattered point, while if $\rho(t)<t$, we say $t$ is a left-scattered point. Points that are right-scattered and left-scattered at the same time will be called isolated points. A point $t \in \mathbb{T}$ such that $t<\sup \mathbb{T}$ and $\sigma(t)=t$, is called a right-dense point. A point $t \in \mathbb{T}$ such that $t>\inf \mathbb{T}$ and $\rho(t)=t$, is called a left-dense point. Points that are right-dense and leftdense at the same time will be called dense points. The set $\mathbb{T}^{k}$ is defined to be $\mathbb{T}^{k}=\mathbb{T} \backslash\{m\}$ if $\mathbb{T}$ has a left-scattered maximum $m$, otherwise $\mathbb{T}^{k}=\mathbb{T}$. The graininess function $\mu: \mathbb{T} \rightarrow[0, \infty)$ is defined by $\mu(t)=\sigma(t)-t$. The notations $[a, b],(a, b)$, and so on, will denote time scale interval such as $[a, b]:=\{t \in \mathbb{T} ; a \leq t \leq b\}$, where $a, b \in \mathbb{T}$. Also, for any $\tau \in \mathbb{T}$, let $\mathbb{T}_{(\tau)}:=[\tau, \infty) \cap \mathbb{T}$ and $\mathbb{T}_{+} ;=\mathbb{T}_{(0)}$.If $t_{0} \in \mathbb{T}$ and $\delta>0$ then we define the following neighborhoods of $t_{0}: U_{\mathbb{T}}\left(t_{0}, \delta\right):=$ $\left(t_{0}-\delta, t_{0}+\delta\right) \cap \mathbb{T}$. Let us consider some examples on time scales see [5, 6].

Example 1. If $h>0, \mathbb{T}=h \mathbb{Z}=\{h k: k \in \mathbb{Z}\}$ is a time scale. Then we have $\sigma(t)=t+h$, and $\rho(t)=t-h$ for all $t \in h \mathbb{Z}$. Hence each point $t \in h \mathbb{Z}$ is a isolated point, and $\mathbb{T}^{k}=\mathbb{T}$.

Example 2. If $\mathbb{T}=\left\{\frac{1}{2 n}, n \in \mathbb{N}\right\} \cup\{0\}$, then we have $\sigma\left(\frac{1}{2}\right)=\frac{1}{2}$, and $\rho\left(\frac{1}{2}\right)=\frac{1}{4}$, i.e., $\frac{1}{2}$ is left scattered. Also, $\sigma\left(\frac{1}{2 n}\right)=\frac{1}{2(n-1)}$ and $\rho\left(\frac{1}{2 n}\right)=$. Therefore, all points $\frac{1}{2 n}, n \in \mathbb{N}, n \geq 2$, are right-scattered and left scattered, i.e., all points $\frac{1}{2 n}, n \in \mathbb{N}, n \geq 2$, are isolated.

Example 3. Let $\mathbb{T}=\mathbb{P}_{1,1}=\cup_{k \in \mathbb{Z}}[2 k, 2 k+1]$. Then

$$
\sigma(t)=\left\{\begin{array}{c}
t+1 \text { if } t \in \cup_{k \in \mathbb{Z}}\{2 k+1\} \\
t \text { if } t \in \cup_{k \in \mathbb{Z}}[2 k, 2 k+1)
\end{array}, \rho(t)=\left\{\begin{array}{c}
t=1 \text { if } t \in \cup_{k \in \mathbb{Z}}\{2 k\} \\
t \text { if } t \in \cup_{k \in \mathbb{Z}}(2 k, 2 k+1]
\end{array}\right.\right.
$$


Definition 4. A function $f: \mathbb{T} \rightarrow \mathbb{R}$ is called regulated if its right sided limits exist (finite) at all right-dense points in $\mathbb{T}$ and its left-sided limits exist (finite) at all left-dense points in $\mathbb{T}$. A function $f: \mathbb{T} \rightarrow \mathbb{R}$ is called rd-continuous if it is continuous at all right-dense points in $\mathbb{T}$ its left-sided limits exist (finite) at all left-dense points in $\mathbb{T}$.

A time scale $\mathbb{T}$ is a nonempty closed subset of $\mathbb{R}$.

Definition 5. Let $f: \mathbb{T} \rightarrow \mathbb{R}$ and $t \in \mathbb{T}^{k}$, We define $f^{\Delta}(t) \in \mathbb{R}$ (provided it exists) with the property that for every $\epsilon>0$ there exists $\delta>0$ such that

$$
\left|f(\sigma(t))-f(s)-f^{\Delta}(t)\right| \sigma(t)-s|| \leq \epsilon|\sigma(t)-s|,
$$

for all $s \in U_{\mathbb{T}}(t, \delta)$. we call $f^{\Delta}(t)$ the delta derivative ( $\Delta$-derivative for short) of all $t_{0}$. Moreover, we say that $f$ is delta-differentiable ( $\Delta$-derivative for short) on $\mathbb{T}^{k}$ provided $f^{\Delta}(t)$ exists for all $t \in \mathbb{T}^{k}$.

We denote by $C_{r d}^{1}\left(\mathbb{T}, \mathbb{R}^{n}\right)$ the set of all functions $f: \mathbb{T} \rightarrow \mathbb{R}^{n}$ that are differentiable on $\mathbb{T}$ and its delta-derivative $f^{\Delta}(t) \in C_{r d}\left(\mathbb{T}, \mathbb{R}^{n}\right)$. The set of rd-continuous (respectively rd-continuous and regressive) functions.

Definition 6. A function $f: \mathbb{T} \rightarrow \mathbb{R}$ is said to be regressive (respectively positively regressive) if $1+\mu(t) f(t) \neq 0$ (respectively $1+\mu(t) f(t)>0$ ) for all $t \in \mathbb{T}^{k}$.

We denote by $\mathscr{R}$ (respectively $\mathscr{R}^{+}$) the set of all regressive (respectively positively regressive) functions from $\mathbb{T}$ to $\mathbb{R}$.

The set $\mathscr{R}$ (respectively $\mathscr{R}^{+}$) of all regressive (respectively positively regressive) functions from $\mathbb{T}$ to $\mathbb{R}$ is an Abelian group with respect to the circle addition operation $\oplus$, given by

$$
(w \oplus q)(t):=w(t)+q(t)+\mu(t) w(t) q(t) .
$$

The inverse element of $w \in \mathscr{R}$ is given by

$$
(\ominus w)(t)=-\frac{w(t)}{1+\mu(t) w(t)},
$$

and so, the circle subtraction operation $\ominus$ is defined by

$$
(w \ominus q)(t)=(w \oplus(\ominus q))(t)=\frac{w(t)-q(t)}{1+\mu(t) q(t)} .
$$

The space of all rd-continuous and regressive functions from $\mathbb{T}$ to $\mathbb{R}$ is denoted by $C_{r d} \mathscr{R}(\mathbb{T}$, $\mathbb{R})$.ectively $\mathscr{R}^{+}$) the set of all regressive (respectively positively regressive) functions from $\mathbb{T}$ to $\mathbb{R}$.

Cosider the following dynamical system on time scales

$$
x^{\Delta}(t)=A_{k}(t)
$$


where $A_{k} \in C_{r d} \mathscr{R}\left(\mathbb{T}_{+}, M_{n}(\mathbb{R})\right), k=1,2, \ldots$, and $t \in\left[t_{k-1}, t_{k}\right) \mathbb{}$. This is a homogenous linear dynamic system on time scales that is nonautonomous, or time-variant. The corresponding nonhomogenous linear dynamic system is given by

$$
x^{\Delta}=A_{k}(t) x+h(t)
$$

where $h \in C_{r d}\left(\mathbb{T}_{+}, \mathbb{R}^{n}\right)$.

A function $x \in C_{r d}^{1}\left(\mathbb{T}_{+}, \mathbb{R}^{n}\right)$ is said to solution of (4) on $\mathbb{T}_{+}$provided $x^{\Delta}(t)=A(t) x(t)+h(t)$ for all $t \in \mathbb{T}_{+}$.

Theorem 7 (Existence and Uniqueness Theorem ([6], Theorem 5.8)). If $A \in C_{r d} \mathscr{R}\left(\mathbb{T}_{+}, M_{n}(\mathbb{R})\right.$ ) and $h \in C_{r d}\left(\mathbb{T}_{+}, \mathbb{R}^{n}\right)$, then for each $(\tau, \eta) \in \mathbb{T}_{+} \times \mathbb{R}^{n}$ the initial value problem

$$
x^{\Delta}=A(t) x+h(t), x(\tau)=\eta,
$$

has a unique solution $x: \mathbb{T}(\tau) \rightarrow \mathbb{R}^{n}$.

A matrix $X_{A_{k}} \in C_{r d} \mathscr{R}\left(\mathbb{T}_{+}, M_{n}(\mathbb{R})\right)$ is said to be matrix solution of (3) if each column of $X_{A_{k}}$ satisfies (3). A fundamental matrix of (3) is a matrix solution $X_{A_{k}}$ of (3) such that $\operatorname{det} X_{A_{k}}(t) \neq 0$ for all $t \in \mathbb{T}_{+}$. A transition matrix of (3) at initial time $\tau \in \mathbb{T}_{+}$is a fundamental matrix such that $X_{A_{k}}(\tau)=I$. The transition matrix of (3) at initial time $\tau \in \mathbb{T}_{+}$is the unique solution of the following matrix initial value problem

$$
X^{\Delta}=A_{k}(t) X, X(\tau)=I,
$$

and $x(t)=\Phi_{A_{k}}(t, \tau) \eta, t \geq \tau$, is the unique solution of the initial value problem

$$
x^{\Delta}=A_{k}(t) x, x(\tau)=\eta .
$$

Lemma 8 ([6], Theorem 5.21). If $A \in C_{r d} \mathscr{R}\left(\mathbb{T}_{+}, M_{n}(\mathbb{R})\right)$ then

(i) $\Phi(t, t)=I$;

(ii) $\Phi_{A}(\sigma(t), s)=(I+\mu(t) A(t)) \Phi_{A}(t, s)$;

(iii) $\Phi_{A}^{-1}(t, s)=\Phi_{\ominus A^{T}}^{T}(t, s)$;

(iv) $\Phi_{A}(t, s)=\Phi_{A}^{-1}(s, t)=\Phi_{\ominus A^{T}}^{T}(s, t)$;

(v) $\Phi_{A}(t, s) \Phi_{A}(s, r)=\Phi_{A}(t, r), t \geq s \geq r$.

Lemma 9 ([6]). If $A \in C_{r d} \mathscr{R}\left(\mathbb{T}_{+}, M_{n}(\mathbb{R})\right)$ and $h \in C_{r d}\left(\mathbb{T}_{+}, \mathbb{R}^{n}\right)$, then for each $(\tau, \eta) \in \mathbb{T}_{+} \times \mathbb{R}^{n}$ the initial value problem

$$
x^{\Delta}=A(t) x+h(t), x(\tau)=\eta,
$$

has a unique solution $x: \mathbb{T}_{(\tau)} \rightarrow \mathbb{R}^{n}$ is given by

$$
x(t)=\Phi_{A}(t, \tau) \eta+\int_{\tau}^{t} \Phi_{A}(t, \sigma(s)) h(s) \Delta s, t \geq \tau .
$$


As in the scaler case, along with (3), consider the following adjoint equation

$$
x^{\Delta}=-A_{k}^{T}(t) x^{\sigma}
$$

If $A_{k} \in C_{r d} \mathscr{R}\left(\mathbb{T}_{+}, M_{n}(\mathbb{R})\right)$ and $h \in C_{r d}\left(\mathbb{T}_{+}, \mathbb{R}^{n}\right)$, then the initial value problem $y^{\Delta}=-A_{k}(t) y^{\sigma}, y(\tau)=\eta$, has a unique solution $x: \mathbb{T}_{(\tau)} \rightarrow \mathbb{R}^{n}$ given by $x(t)=\Phi_{\ominus A_{k}^{T}}^{T}(t, \tau), t \geq \tau$.

Lemma 10 ([6], Theorem 5.27). If $A \in C_{r d} \mathscr{R}\left(\mathbb{T}_{+}, M_{n}(\mathbb{R})\right)$ and $h \in C_{r d}\left(\mathbb{T}_{+}, \mathbb{R}^{n}\right)$, then for each $(\tau, \eta) \in \mathbb{T}_{+} \times \mathbb{R}^{n}$ the initial value problem

$$
x^{\Delta}=-A^{T}(t) x^{\sigma}+h(t), x(\tau)=\eta
$$

has a unique solution $x: \mathbb{T}_{(\tau)} \rightarrow \mathbb{R}^{n}$ is given by

$$
x(t)=\Phi_{\ominus A^{T}}(t, \tau) \eta+\int_{\tau}^{t} \Phi_{\ominus A^{T}}(t, s) h(s) \Delta s, t \in \mathbb{T}_{(\tau)} .
$$

Proposition 11. For the system (5) with $A_{k} \in M_{n}(\mathbb{R})$ constant, there exist scalar functions $\gamma_{0}(t, \tau), \ldots, \gamma_{n-1}(t, \tau) \in C_{r d}^{\infty}\left(\mathbb{T}_{+}, \mathbb{R}\right)$ such that the unique solution has representations

$$
e_{A_{k}^{T}}(t, \tau)=\sum_{j=0}^{n-1} \gamma_{j}(t, \tau)\left(A_{k}^{T}\right)^{j}
$$

\section{Controllability}

Consider the following linear time-varying impulsive adjoint dynamic system

$$
\left\{\begin{array}{l}
x^{\Delta}(t)=-A_{k}^{T}(t) x^{\sigma}(t)+B_{k}(t) u(t), t \in\left[t_{k-1}, t_{k}\right) \mathbb{T} \\
x\left(t_{k}^{+}\right)=\left(1+c_{k}\right) x\left(t_{k}\right), t=t_{k}, k=1,2, \ldots \\
x\left(t_{0}\right)=x_{0}
\end{array}\right.
$$

with the following conditions:

(i) Time scale $\mathbb{T}$ is unbounded above with bounded graininess(i.e. $\sup \mathbb{T}=\infty$ and $\mu(t)<$ $\infty)$.

(ii) $t_{0}<t_{1}<t_{2}<\cdots t_{k}<\cdots$, with $\lim _{k \rightarrow \infty} t_{k}=\infty$, where $t_{k} \in \mathbb{T}_{+}$are right-dense.

(iii) $x\left(t_{k}^{+}\right):=\lim _{h \rightarrow 0^{+}} x\left(t_{k}+h\right), v\left(t_{k}^{-}\right):=\lim _{h \rightarrow 0^{+}} x\left(t_{k}-h\right)$ and $c_{k} \in \mathbb{R}$ are constants.

(iv) $A_{k}(\cdot) \in C_{r d} \mathscr{R}\left(\mathbb{T}_{+}, M_{n}(\mathbb{R})\right), B_{k}(\cdot) \in C_{r d} \mathscr{R}\left(\mathbb{T}_{+}, M_{n \times m}(\mathbb{R})\right), x \in \mathbb{R}^{n}$ is the state variable and $u \in \mathbb{R}^{m}$ is the control input. 
Lemma 12. For any $t \in\left[t_{k-1}, t_{k}\right)_{\mathbb{T}}, k=1,2,3, \cdots$, the solution of intial value problem (7) is given by

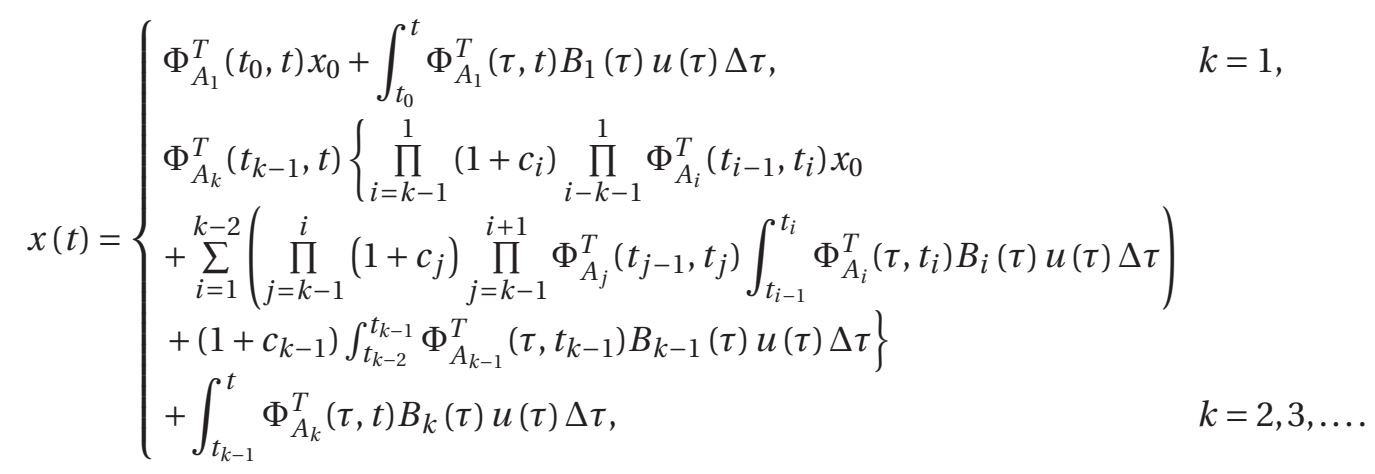

Definition 13. The impulsive system (7) is said to be completely controllable on $\left[t_{0}, t_{f}\right]_{\mathbb{T}}$ if given initial state $x_{0} \in \mathbb{R}^{n}$, there exists a picecewise rd-continuous input signal $u(\cdot):\left[t_{0}, t_{f}\right]_{\mathbb{T}} \rightarrow$ $\mathbb{R}^{m}$ such that the corresponding solution of (7) satisfies $x\left(t_{f}\right)=0$.

We consider the following matrices.

For $t \in\left[t_{0}, t_{1}\right]_{\mathbb{T}}$

$$
G_{1}:=G_{1}\left(t_{0}, t_{f}, t_{f}\right)=\int_{t_{0}}^{t_{f}} \Phi_{A_{1}}^{T}\left(\tau, t_{f}\right) B_{1}(\tau) B_{1}^{T}(\tau) \Phi_{A_{1}}\left(\tau, t_{f}\right) \Delta \tau,
$$

for $t \in\left[t_{l-1}, t_{l}\right)_{\mathbb{T}}$, where $2 \leq l \leq k-1$

$$
G_{l}:=G_{l}\left(t_{l-1}, t_{l}, t_{f}\right)=\int_{t_{l-1}}^{t_{f}} \Phi_{A_{l}}^{T}\left(\tau, t_{f}\right) B_{l}(\tau) B_{l}^{T}(\tau) \Phi_{A_{l}}\left(\tau, t_{f}\right) \Delta \tau,
$$

and for $t \in\left[t_{k-1}, t_{k}\right) \mathbb{T}$

$$
G_{k}:=G_{k}\left(t_{k-1}, t_{f}, t_{f}\right)=\int_{t_{k-1}}^{t_{f}} \Phi_{A_{k}}^{T}\left(\tau, t_{f}\right) B_{k}(\tau) B_{k}^{T}(\tau) \Phi_{A_{k}}\left(\tau, t_{f}\right) \Delta \tau .
$$

\section{Theorem 14.}

(i) If there exist at least $l \in\{1,2, \cdots, k\}$ such that

$$
\operatorname{rank}\left(G_{l}\left(t_{l-1}, t_{l}, t_{f}\right)\right)=n,
$$

then the impulsive system (7) is controllable on $\left[t_{0}, t_{f}\right]_{\mathbb{T}}\left(t_{f} \in\left[t_{l-1}, t_{l}\right)_{\mathbb{T}}\right)$.

(ii) Assume that $c_{i} \neq-1, i=1,2, \cdots, k$. If impulsive system (7) is controllable on $\left[t_{0}, t_{f}\right]_{\mathbb{T}}\left(t_{f} \in\left[t_{l-1}, t_{l}\right)_{\mathbb{T}}\right)$, then

$$
\operatorname{rank}\left(G_{1} \cdots G_{k}\right)=n
$$


Proof. Let $l \in\{1,2, \cdots, k\}$ such that the $\operatorname{rank}_{l}\left(t_{l-1}, t_{l}, t_{f}\right)=n$, that is $G_{l}\left(t_{l-1}, t_{l}, t_{f}\right)$ is ivertible. Then for a given $x_{0} \in \mathbb{R}^{n}$, choose a control function given as:

$$
u(t)= \begin{cases}-B_{1}^{T}(t) \Phi_{A_{1}}\left(t, t_{f}\right) G_{1}^{-1} \Phi_{A_{1}}^{T}\left(t_{0}, t_{f}\right) x_{0}, & \text { for } t \in\left[t_{0}, t_{1}\right]_{\mathbb{T}}, 2 \leq l \leq k-1, \\ -B_{l}^{T}(t) \Phi_{A_{l}}\left(t, t_{f}\right) G_{l}^{-1} \Phi_{A_{l}}^{T}\left(t_{l-1}, t_{f}\right) \prod_{i=l-1}^{1}\left(1+c_{i}\right) & \\ \times \prod_{i=l-1}^{1} \Phi_{A_{i}}^{T}\left(t_{i-1}, t_{i}\right) x_{0}, & \text { for } t \in\left[t_{l-1}, t_{l}\right)_{\mathbb{T}}, \\ 0 & \text { if } t \in\left[t_{0}, t_{f}\right] \backslash\left[t_{l-1}, t_{l}\right), \\ -B_{k}^{T}(t) \Phi_{k}\left(t, t_{f}\right) G_{k}^{-1} \Phi_{A_{k}}^{T}\left(t_{k-1}, t_{f}\right) \prod_{i=k-1}^{1}\left(1+c_{i}\right) & \\ \times \prod_{i=k-1}^{1} \Phi_{A_{i}}^{T}\left(t_{i-1}, t_{i}\right) x_{0}, & \text { for } t \in\left[t_{k-1}, t_{k}\right)_{\mathbb{T}} \\ 0 & \text { if } t \in\left[t_{0}, t_{f}\right]_{\mathbb{T}} \backslash\left[t_{k-1}, t_{k}\right)_{\mathbb{T}} .\end{cases}
$$

Obviously, the control input $u(\cdot)$ is piecewise rd-continuous on $\left[t_{0}, t_{f}\right]_{\mathbb{T}}$. By Lemma (12), we have

$$
x\left(t_{f}\right)=\Phi_{A_{1}}^{T}\left(t_{0}, t_{f}\right) x_{0}-\int_{t_{0}}^{t_{f}} \Phi_{A_{1}}^{T}\left(\tau, t_{f}\right) B_{1}(\tau) B_{1}^{T}(\tau) \Phi_{A_{1}}\left(\tau, t_{f}\right) G_{1}^{-1} \Phi_{A_{1}}^{T}\left(t_{0}, t_{f}\right) x_{0} \Delta \tau .
$$

By using equation (9)

$$
\begin{aligned}
x\left(t_{f}\right) & =\Phi_{A_{1}}^{T}\left(t_{0}, t_{f}\right) x_{0}-G_{1} G_{1}^{-1} \Phi_{A_{1}}^{T}\left(t_{0}, t_{f}\right) x_{0} \\
& =0, \text { for } t \in\left[t_{0}, t_{1}\right]_{\mathbb{J}} .
\end{aligned}
$$

Thus the system (7) is controllable on $\left[t_{0}, t_{1}\right]_{\mathbb{T}}$.

Similarly, for $2 \leq l \leq k-1$ and $t \in\left[t_{l-1}, t_{l}\right)_{\mathbb{T}}$

$$
\begin{aligned}
x\left(t_{f}\right)= & \Phi_{A_{l}}^{T}\left(t_{l-1}, t_{f}\right) \prod_{i=l-1}^{1}\left(1+c_{i}\right) \prod_{i=l-1}^{1} \Phi_{A_{i}}^{T}\left(t_{i-1}, t_{i}\right) x_{0} \\
& -\int_{t_{l-1}}^{t} \Phi_{A_{l}}^{T}\left(\tau, t_{f}\right) B_{l}(\tau) B_{l}^{T}(t) \Phi_{A_{l}}\left(t, t_{f}\right) G_{l}^{-1} \Phi_{A_{l}}^{T}\left(t_{l-1}, t_{f}\right) \prod_{i=l-1}^{1}\left(1+c_{i}\right) \prod_{i=l-1}^{1} \Phi_{A_{i}}^{T}\left(t_{i-1}, t_{i}\right) x_{0} \Delta \tau
\end{aligned}
$$

it follows from equation (10)

$$
x\left(t_{f}\right)=0, t \in\left[t_{l-1}, t_{l}\right)_{\mathbb{T}},
$$

and similarly, we obtain

$$
x\left(t_{f}\right)=0, t \in\left[t_{k-1}, t_{k}\right) \mathbb{T} .
$$

Thus the system (7) is controllable on $\left[t_{0}, t_{f}\right]_{\mathbb{T}}$. So (i) holds.

(ii) Assume that (7) is controllable on $\left[t_{0}, t_{f}\right]_{\mathbb{T}}$. we have to prove that

$$
\operatorname{rank}\left(G_{1} \cdots G_{k}\right)=n \text {. }
$$


Suppose contrary that

$$
\operatorname{rank}\left(G_{1} \cdots G_{k}\right)<n,
$$

then, there exist a nonzero $x_{\alpha} \neq 0 \in \mathbb{R}^{n}$ such that

$$
x_{\alpha}^{T} G_{i}\left(t_{i-1}, t_{i}, t_{f}\right) x_{\alpha}=0, i=1,2, \cdots, k .
$$

for $i=1$

$$
x_{\alpha}^{T} G_{1} x_{\alpha}=\int_{t_{0}}^{t_{f}} x_{\alpha}^{T} \Phi_{A_{1}}^{T}\left(\tau, t_{f}\right) B_{1}(\tau) B_{1}^{T}(\tau) \Phi_{A_{1}}\left(\tau, t_{f}\right) x_{\alpha} \Delta \tau,
$$

As $x_{\alpha}^{T} \Phi_{A_{1}}^{T}\left(t, t_{f}\right) B_{1}(t)$ is rd-continuous functions so

$$
\left\|x_{\alpha}^{T} \Phi_{A_{1}}^{T}\left(t, t_{f}\right) B_{1}(t)\right\|^{2}=0,
$$

which follows that

$$
B_{1}^{T}(t) \Phi_{A_{1}}^{T}\left(t, t_{f}\right) x_{\alpha}=0, t \in\left[t_{0}, t_{1}\right]_{\mathbb{T}},
$$

For $l=2, \cdots, k-1$,

$$
\begin{gathered}
x_{\alpha}^{T} G_{l} x_{\alpha}=\int_{t_{l-1}}^{t_{f}} x_{\alpha}^{T} \Phi_{A_{l}}^{T}\left(\tau, t_{f}\right) B_{l}(\tau) B_{l}^{T}(\tau) \Phi_{A_{l}}\left(\tau, t_{f}\right) x_{\alpha} \Delta \tau=0, \\
x_{\alpha}^{T} \Phi_{A_{l}}^{T}\left(t, t_{f}\right) B_{l}(t) B_{l}^{T}(t) \Phi_{A_{l}}\left(t, t_{f}\right) x_{\alpha}=\left\|x_{\alpha}^{T} \Phi_{l}\left(t, t_{f}\right) B_{l}(\tau)\right\|^{2}, \\
B_{l}^{T}(t) \Phi_{A_{l}}^{T}\left(t, t_{f}\right) x_{\alpha}=0, t \in\left[t_{l-1}, t_{l}\right) \mathbb{\mathbb { }} .
\end{gathered}
$$

Similarly,

$$
B_{k}^{T}(t) \Phi_{A_{k}}^{T}\left(t, t_{f}\right) x_{\alpha}=0, t \in\left[t_{k-1}, t_{k}\right) \mathbb{\pi} .
$$

However, the impulsive system (7) is controllable on $\left[t_{0}, t_{f}\right]_{\mathbb{T}}$, and so choosing $x_{0}=x_{\alpha}$, there exists a piecewise rd-continuous input $u(\cdot)$ such that

$$
0=x\left(t_{f}\right)=\Phi_{A_{1}}^{T}\left(t_{0}, t_{f}\right) x_{\alpha}+\int_{t_{0}}^{t_{f}} \Phi_{A_{1}}^{T}\left(\tau, t_{f}\right) B_{1}(\tau) u(\tau) \Delta \tau ; k=1 .
$$

Multiply through by $x_{\alpha}^{T}$ in (16) and using the transpose of equation (13), we obtain

$$
\Phi_{A_{1}}^{T}\left(t_{0}, t_{f}\right) x_{\alpha}^{T} x_{\alpha}=0
$$

Similarly,

$$
\begin{aligned}
x\left(t_{f}\right)= & \Phi_{A_{k}}^{T}\left(t_{k-1}, t_{f}\right)\left\{\prod_{i=k-1}^{1}\left(1+c_{i}\right) \prod_{i=k-1}^{1} \Phi_{A_{i}}^{T}\left(t_{i-1}, t_{i}\right) x_{\alpha}\right. \\
& +\sum_{i=1}^{k-2}\left(\prod_{j=k-1}^{i}\left(1+c_{j}\right) \prod_{j=k-1}^{i+1} \Phi_{A_{j}}^{T}\left(t_{j-1}, t_{j}\right) \times \int_{t_{i-1}}^{t_{i}} \Phi_{A_{i}}^{T}\left(\tau, t_{i}\right) B_{i}(\tau) u(\tau) \Delta \tau\right)
\end{aligned}
$$




$$
\begin{aligned}
& \left.+\left(1+c_{k-1}\right) \int_{t_{k-2}}^{t_{k-1}} \Phi_{A_{k-1}}^{T}\left(\tau, t_{k-1}\right) B_{k-1}(\tau) u(\tau) \Delta \tau\right\} \\
& +\int_{t_{k-1}}^{t_{f}} \Phi_{A_{k}}^{T}\left(\tau, t_{f}\right) B_{k}(\tau) u(\tau) \Delta \tau, \quad k=2,3, \ldots
\end{aligned}
$$

Multiplying by $\Phi_{A_{1}}^{T}\left(t_{1}, t_{2}\right) \Phi_{A_{2}}^{T}\left(t_{2}, t_{3}\right) \cdots \Phi_{A_{k}}^{T}\left(t_{k-1}, t_{f}\right)$ and $x_{\alpha}^{T}$ in (18), and using equations (14), and (15), we obtain

$$
\prod_{j=2}^{k}\left(1+c_{j}\right) x_{\alpha}^{T} x_{\alpha}=0
$$

From equations (17) and (19), it follows that $x_{\alpha}^{T} x_{\alpha}=0$. This contradicts $x_{\alpha} \neq 0$ and so, we conclude that

$$
\operatorname{rank}\left(G_{1} \cdots G_{k}\right)=n
$$

Our next result give us necessary and sufficient conditions for time invariant system. Let us define new matrices:

$$
W_{i}=\left[B_{i}^{T} B_{i}^{T} A_{i} \cdots B_{i}^{T} A_{i}^{n-1}\right], i=1,2, \cdots, k .
$$

Theorem 15. Assume that $c_{i} \neq-1, i=1,2, \ldots, k$, and $A_{k}(t)=A_{k}, B_{k}(t)=B_{k}$ are constant matrices. Then, the impulsive system (7) is controllable on $\left[t_{0}, t_{f}\right]_{\mathbb{T}}\left(t_{f} \in\left[t_{k-1}, t_{k}\right)_{\mathbb{T}}\right)$, if and only if

$$
\operatorname{rank}\left(W_{1} W_{2} \cdots W_{k}\right)=n
$$

Proof. Suppose that the impulsive system (7) is controllable on $\left[t_{0}, t_{f}\right]_{\mathbb{T}}$. If the rank condition (20) does not hold, then there exist $x_{\alpha} \in \mathbb{R}^{n}$ with $x_{\alpha} \neq 0$, such that

$$
B_{i} A_{i}^{j} x_{\alpha}=0
$$

for $i=1, \cdots, k, j=0,1, \ldots, n-1$.

Consider

$$
G_{1}\left(t_{0}, t_{f}, t_{f}\right) x_{\alpha}=\int_{t_{0}}^{t_{f}} e_{A_{1}}^{T}\left(\tau, t_{f}\right) B_{1} B_{1}^{T} e_{A_{1}}\left(\tau, t_{f}\right) x_{\alpha} \Delta \tau .
$$

Using Proposition (11), and equation (21), we obtain

$$
\begin{aligned}
G_{1}\left(t_{0}, t_{f}, t_{f}\right) x_{\alpha} & =\int_{t_{0}}^{t_{f}} e_{A_{1}}^{T}\left(\tau, t_{f}\right) B_{1} B_{1}^{T} \sum_{j=0}^{n-1} \gamma_{1 j}\left(\tau, t_{f}\right) A_{1}^{j} x_{\alpha} \Delta \tau, \\
& =\int_{t_{0}}^{t_{f}} e_{A_{1}}^{T}\left(\tau, t_{f}\right) B_{1} \sum_{j=0}^{n-1} \gamma_{1 j}\left(\tau, t_{f}\right) B_{1}^{T} A_{1}^{j} x_{\alpha} \Delta \tau \\
& =0 .
\end{aligned}
$$


By again using Proposition (11), and equation (21), it follows that

$$
\begin{aligned}
G_{l}\left(t_{l-1}, t_{l}, t_{f}\right) x_{\alpha} & =\int_{t_{l-1}}^{t_{f}} e_{A_{l}}^{T}\left(\tau, t_{f}\right) B_{l} B_{l}^{T} e_{A_{l}}\left(\tau, t_{f}\right) x_{\alpha} \Delta \tau \\
& =\int_{t_{l-1}}^{t_{f}} e_{A_{l}}^{T}\left(\tau, t_{f}\right) B_{l} B_{l}^{T} \sum_{j=0}^{n-1} \gamma_{l j}\left(\tau, t_{f}\right) A_{l}^{j} x_{\alpha} \Delta \tau \\
& =\int_{t_{l-1}}^{t_{f}} e_{A_{l}}^{T}\left(\tau, t_{f}\right) B_{l} \sum_{j=0}^{n-1} \gamma_{l j}\left(\tau, t_{f}\right) B_{l}^{T} A_{l}^{j} x_{\alpha} \Delta \tau \\
& =0,
\end{aligned}
$$

for $2 \leq l \leq k-1$. Similarly, $G_{k}\left(t_{k-1}, t_{k}, t_{f}\right) x_{\alpha}=0$, which follows that

$$
\operatorname{rank}\left(G_{0} G_{1} \cdots G_{k}\right)<n
$$

This contradicts the conclusion (ii) of Theorem 14 and therefore, we can conclude that the condition (20) is true.

Conversely, suppose that (20) holds. If the impulsive system (7) is not controllable on $\left[t_{0}, t_{f}\right]_{\mathbb{T}}\left(t_{f} \in\left[t_{k-1}, t_{k}\right)_{\mathbb{T}}\right)$, then it follows from the conclusion (i) of Theorem 14 that the matrices $G_{1}\left(t_{0}, t_{f}, t_{f}\right), G_{l}\left(t_{l-1}, t_{l}, t_{f}\right)$ and $G_{k}\left(t_{k-1}, t_{k}, t_{f}\right)$ are not invertible. Thus, there exist $x_{\alpha} \in \mathbb{R}^{n}$ with $x_{\alpha} \neq 0$, such that

$$
\begin{aligned}
x_{\alpha}^{T} G_{1}\left(t_{0}, t_{f}, t_{f}\right) x_{\alpha} & =\int_{t_{0}}^{t_{f}} x_{\alpha}^{T} e_{A_{1}}^{T}\left(\tau, t_{f}\right) B_{1} B_{1}^{T} e_{A_{1}}\left(\tau, t_{f}\right) x_{\alpha} \Delta \tau=0, \\
x_{\alpha}^{T} G_{l}\left(t_{l-1}, t_{l}, t_{f}\right) x_{\alpha} & =\int_{t_{0}}^{t_{f}} x_{\alpha}^{T} e_{A_{l}}^{T}\left(\tau, t_{f}\right) B_{l} B_{l}^{T} e_{A_{l}}\left(\tau, t_{f}\right) x_{\alpha} \Delta \tau=0,2 \leq l \leq k-1, \\
x_{\alpha}^{T} G_{k}\left(t_{k-1}, t_{k}, t_{f}\right) x_{\alpha} & =\int_{t_{0}}^{t_{f}} x_{\alpha}^{T} e_{A_{k}}^{T}\left(\tau, t_{f}\right) B_{k} B_{k}^{T} e_{A_{k}}\left(\tau, t_{f}\right) x_{\alpha} \Delta \tau=0 .
\end{aligned}
$$

Exactly as in proof of Theorem 14, it follows that

$$
\begin{array}{ll}
B_{1}^{T} e_{A_{1}}\left(t, t_{f}\right) x_{\alpha}=0, & \text { for } t \in\left[t_{0}, t_{1}\right]_{\mathbb{T}}, \\
B_{l}^{T} e_{A_{l}}\left(t, t_{f}\right) x_{\alpha}=0, & \text { for } t \in\left[t_{l-1}, t_{l}\right)_{\mathbb{T}},
\end{array}
$$

where $2 \leq l \leq k-1$, and

$$
B_{k}^{T} e_{A_{k}}\left(t, t_{f}\right) x_{\alpha}=0, \quad \text {, for } t \in\left[t_{k-1}, t_{k}\right)_{\mathbb{T}}
$$

Differentiating equations (22), (23) and (24) $j$ times, where $(0 \leq j \leq n-1)$, we have

$$
\begin{array}{ll}
B_{1}^{T} A_{1}^{j} e_{A_{1}}\left(\tau, t_{f}\right) x_{\alpha}=0, & \text { for } t \in\left[t_{0}, t_{1}\right]_{\mathbb{T}}, \\
B_{l}^{T} A_{l}^{j} e_{A_{l}}\left(t, t_{f}\right) x_{\alpha}=0, & \text { for } t \in\left[t_{l-1}, t_{l}\right)_{\mathbb{T}},
\end{array}
$$


where $2 \leq l \leq k-1$, and

$$
B_{k}^{T} A_{k}^{j} e_{A_{k}}\left(t, t_{f}\right) x_{\alpha}=0 \text {, for } t \in\left[t_{k-1}, t_{k}\right)_{\mathbb{T}} \text {. }
$$

If we take $t=t_{f}$ in equations (25), (26) and (27), then it follows that $B_{i}^{T} A_{i}^{j} x_{\alpha}=0$, for $i=1, \cdots, k$, and $j=0, \cdots, n-1$. Which implies that the rank condition (20) fails, which gives contradiction. So the impulsive system (7) ic controllable on $\left[t_{0}, t_{f}\right]_{\mathbb{T}}\left(t_{f} \in\left[t_{k-1}, t_{k}\right)_{\mathbb{T}}\right)$.

\section{Observability}

In this section we have to study the observability properties for the following linear timevarying impulsive dynamic system:

$$
\left\{\begin{array}{rlrl}
x^{\Delta}(t) & =-A_{k}(t) x^{\sigma}(t)+B_{k}(t) u(t), & t \in\left[t_{k-1}, t_{k}\right) \mathbb{T} \\
x\left(t_{k}^{+}\right) & =\left(1+c_{k}\right) x\left(t_{k}\right), & t=t_{k}, k=1,2, \ldots, \\
y(t) & =C_{k}(t) x(t)+D_{k}(t) u(t), & \\
x\left(t_{0}\right) & =x_{0}
\end{array}\right.
$$

under the following conditions:

(i) Time scale $\mathbb{T}$ is unbounded above with bounded graininess (i.e. $\sup \mathbb{\mathbb { T }}=\infty$ and $\mu(t)<$ $\infty)$.

(ii) $t_{0}<t_{1}<t_{2}<\cdots t_{k}<\cdots$, with $\lim _{k \rightarrow \infty} t_{k}=\infty$. where, $t_{k} \in \mathbb{T}_{+}$are right-dense.

(iii) $x\left(t_{k}^{+}\right):=\lim _{h \rightarrow 0^{+}} x\left(t_{k}+h\right), x\left(t_{k}^{-}\right):=\lim _{h \rightarrow 0^{+}} x\left(t_{k}-h\right)$ and $c_{k} \in \mathbb{R}$ are constants.

(iv) $A_{k}(\cdot) \in C_{r d} \mathscr{R}\left(\mathbb{T}_{+}, M_{n}(\mathbb{R})\right), B_{k}(\cdot) \in C_{r d} \mathscr{R}\left(\mathbb{T}_{+}, M_{n \times m}(\mathbb{R})\right), x \in \mathbb{R}^{n}$ is the state variable and $u \in \mathbb{R}^{m}$ is the control input.

(v) $C_{k}(\cdot) \in C_{r d} \mathscr{R}\left(\mathbb{T}_{+}, M_{p \times n}(\mathbb{R})\right), D_{k}(\cdot) \in C_{r d} \mathscr{R}\left(\mathbb{T}_{+}, M_{p \times m}(\mathbb{R})\right), y \in \mathbb{R}^{p}$ is the output.

Complete observability of linear impulsive systems is define as follows:

Definition 16. The impulsive system (28) is said to be completely observable on $\left[t_{0}, t_{f}\right]_{\mathbb{T}}\left(t_{f}>t_{0}\right)$ if any initial state $x\left(t_{0}\right)=x_{0} \in \mathbb{R}^{n}$ is uniquely determined by the corresponding system input $u(t)$ and the system output $y(t)$ for $\left[t_{0}, t_{f}\right]_{\mathbb{T}}$.

Theorem 17. Assume that $1+c_{i} \geq 0, i=1,2, \ldots, k$. Then, the impulsive system (28) is observable on $\left[t_{0}, t_{f}\right]_{\mathbb{T}}\left(t_{f} \in\left(t_{k}, t_{k+1}\right]\right) \mathbb{\mathbb { T }}$ if and only if the matrix

$$
M\left(t_{0}, t_{f}\right):=M\left(t_{0}, t_{0}, t_{1}\right)+\sum_{i=2}^{k-1} \prod_{j=1}^{i}\left(1+c_{j}\right) M\left(t_{0}, t_{i-1}, t_{i}\right)+\prod_{j=1}^{k}\left(1+c_{j}\right) M\left(t_{0}, t_{k-1}, t_{f}\right)
$$

is invertible, where

$$
M\left(t_{0}, t_{0}, t_{1}\right):=\int_{t_{0}}^{t_{1}} \Phi_{A_{1}}\left(t_{0}, \tau\right) C_{1}^{T}(\tau) C_{1}(\tau) \Phi_{A_{1}}^{T}\left(t_{0}, \tau\right) \Delta \tau,
$$


and

$$
M\left(t_{0}, t_{i-1}, t_{i}\right):=\int_{t_{i-1}}^{t_{i}} \Omega_{i}\left(t_{0}, \tau\right) C_{i}^{T}(\tau) C_{i}(\tau) \Omega_{i}^{T}\left(t_{0}, \tau\right) \Delta \tau i=2, \ldots, k-1,
$$

with

$$
M\left(t_{0}, t_{k-1}, t_{f}\right)=\int_{t_{k-1}}^{t_{f}} \Omega_{k}\left(t_{0}, \tau\right) C_{k}^{T}(\tau) C_{k}(\tau) \Omega_{k}^{T}\left(t_{0}, \tau\right) \Delta \tau
$$

$$
\Omega_{i}^{T}\left(t_{0}, \tau\right)=\Phi_{A_{i}}^{T}\left(t_{i-1}, \tau\right) \Phi_{A_{i-1}}^{T}\left(t_{i-2}, t_{i-1}\right) \cdots \Phi_{A_{1}}^{T}\left(t_{0}, t_{1}\right), \quad i=1, \ldots, k .
$$

Proof. Suppose $M\left(t_{0}, t_{f}\right)_{\mathbb{T}}$ is invertible. From (8) and (28), we obtain

$$
y(t)=C_{1}(t) \Phi_{A_{1}}^{T}\left(t_{0}, t\right) x_{0}+C_{1}(t) \int_{t_{0}}^{t_{1}} \Phi_{A_{1}}^{T}(\tau, t) B_{1}(\tau) u(\tau) \Delta \tau+D_{1}(t) u(t), t \in\left[t_{0}, t_{1}\right]_{\mathbb{T}}
$$

and

$$
\begin{aligned}
y(t)= & C_{l}(t) \Phi_{A_{l}}^{T}\left(t_{l-1}, t\right)\left\{\prod_{i=l-1}^{1}\left(1+c_{i}\right) \prod_{i=l-1}^{1} \Phi_{A_{i}}^{T}\left(t_{i-1}, t_{i}\right) x_{0}\right. \\
& C_{l}(t)+\sum_{i=1}^{l-2}\left(\prod_{j=l-1}^{i}\left(1+c_{j}\right) \prod_{j=l-1}^{i+1} \Phi_{A_{j}}^{T}\left(t_{j-1}, t_{j}\right) \int_{t_{i-1}}^{t_{i}} \Phi_{A_{i}}^{T}\left(\tau, t_{i}\right) B_{i}(\tau) u(t) \Delta \tau\right) \\
& \left.C_{l}(t)+\left(1+c_{k-1}\right) \int_{t_{l-2}}^{t_{l-1}} \Phi_{A_{l-1}}^{T}\left(\tau, t_{l-1}\right) B_{l-1}(\tau) u(t) \Delta \tau\right\} \\
& C_{l}(t)+\int_{t_{l-1}}^{t} \Phi_{A_{l}}^{T}(\tau, t) B_{l}(\tau) u(t) \Delta \tau+D_{l}(\tau) u(\tau), \text { for } t \in\left(t_{l-1}, t_{l}\right]_{\mathbb{T}}, l=2, \ldots, k .
\end{aligned}
$$

It is easy from the Definition 16 that the observability of system (28) is equivalent to the observability of $y(t)$ is given by

$$
y(t)=\left\{\begin{array}{l}
C_{1}(t) \Phi_{A_{1}}^{T}\left(t_{0}, t\right) x_{0} t \in\left[t_{0}, t_{1}\right]_{\mathbb{T}} \\
\prod_{i=l-1}^{1}\left(1+c_{i}\right) C_{l}(t) \Omega_{l}^{T}\left(t_{0}, t\right) x_{0} t \in\left(t_{l-1}, t_{l}\right]_{\mathbb{T}}, \quad l=2, \ldots, k,
\end{array}\right.
$$

as $u(t)=0$. Now, multiply by $\Omega_{l}\left(t_{0}, t\right) C_{l}^{T}(t)$ to both sides of (31) and integrating with respect to $t_{0}$ to $t_{f}$, we have

$$
\begin{aligned}
& \int_{t_{0}}^{t_{f}} \Omega_{l}\left(t_{0}, \tau\right) C_{l}^{T}(\tau) y(\tau) \Delta \tau \\
& =\left[\int_{t_{0}}^{t_{1}} \Phi_{A_{1}}\left(t_{0}, \tau\right) C_{1}^{T}(\tau) C_{1}(\tau) \Phi_{A_{1}}^{T}\left(t_{0}, \tau\right) \Delta \tau+\sum_{i=2}^{k-1} \prod_{j=i}^{i}\left(1+c_{j}\right) \int_{t_{i-1}}^{t_{i}} \Omega_{i}\left(t_{0}, \tau\right) C_{i}^{T}(\tau) C_{i}(\tau) \Omega_{i}^{T}\left(t_{0}, \tau\right) \Delta \tau\right. \\
& \left.\quad+\prod_{j=1}^{k}\left(1+c_{j}\right) \int_{t_{k-1}}^{t_{f}} \Omega_{k}\left(t_{0}, \tau\right) C_{k}^{T}(\tau) C_{k}(\tau) \Omega_{k}^{T}\left(t_{0}, \tau\right) \Delta \tau\right] x_{0}
\end{aligned}
$$

and so,

$$
\int_{t_{0}}^{t_{f}} \Omega_{l}\left(t_{0}, \tau\right) C_{l}^{T}(\tau) y(\tau) \Delta \tau
$$




$$
\begin{aligned}
& =\left[M\left(t_{0}, t_{0}, t_{1}\right)+\sum_{i=2}^{k-1} \prod_{j=1}^{i}\left(1+c_{j}\right) M\left(t_{0}, t_{i-1}, t_{i}\right)+\prod_{j=1}^{k}\left(1+c_{j}\right) M\left(t_{0}, t_{k-1}, t_{f}\right)\right] x_{0} \\
& =M\left(t_{0}, t_{f}\right) x_{0} .
\end{aligned}
$$

Obviously, the left hand side of equation (32) depends on $y(t), t \in\left[t_{0}, t_{f}\right]_{\mathbb{T}}$. Since the matrix $M\left(t_{0}, t_{f}\right)$ is invertible, then from linear algebraic equation (32) we deduce that $x\left(t_{0}\right)=x_{0}$ is uniquely determined by the corresponding system output $y(t)$ for $t \in\left[t_{0}, t_{f}\right]_{\mathbb{T}}$.

Conversely, suppose that the matrix $M\left(t_{0}, t_{f}\right)$ is not invertible, then there exist a nonzero $x_{\alpha} \in \mathbb{R}^{n}$, such that

$$
x_{\alpha}^{T} M\left(t_{0}, t_{f}\right) x_{\alpha}=0 .
$$

Since $1+c_{i} \geq 0, i=1,2, \ldots, k, M\left(t_{0}, t_{0}, t_{1}\right), M\left(t_{0}, t_{i-1}, t_{i}\right)$ for $i=2 \cdots, k-1$ and $M\left(t_{0}, t_{k-1}, t_{f}\right)$ are positive semidefinite matrics, we have

$$
\begin{aligned}
& x_{\alpha}^{T} M\left(t_{0}, t_{0}, t_{1}\right) x_{\alpha}=0, \\
& x_{\alpha}^{T} M\left(t_{0}, t_{i-1}, t_{i}\right) x_{\alpha}=0, \quad \text { for } i=2, \ldots, k-1 \\
& x_{\alpha}^{T} M\left(t_{0}, t_{k-1}, t_{f}\right) x_{\alpha}=0 .
\end{aligned}
$$

Choose $x_{0}=x_{\alpha}$. Then, from (31) and (33), it follows that

$$
\begin{aligned}
\int_{t_{0}}^{t_{f}} y^{T}(\tau) y(\tau) \Delta \tau= & \int_{t_{0}}^{t_{1}} x_{\alpha}^{T} \Phi_{A_{1}}\left(t_{0}, \tau\right) C_{1}^{T}(\tau) C_{1}(\tau) \Phi_{A_{1}}^{T}\left(t_{0}, \tau\right) x_{\alpha} \Delta \tau \\
& +\sum_{i=2}^{k-1}\left[\prod_{j=1}^{i}\left(1+c_{j}\right)\right]^{2} \int_{t_{i-1}}^{t_{i}} x_{\alpha}^{T} \Omega_{i}\left(t_{0}, \tau\right) C_{i}^{T}(\tau) C_{i}(\tau) \Omega_{i}^{T}\left(t_{0}, \tau\right) x_{\alpha} \Delta \tau \\
& +\left[\prod_{j=1}^{k}\left(1+c_{j}\right)\right]^{2} \int_{t_{k-1}}^{t_{f}} x_{\alpha}^{T} \Omega_{k}\left(t_{0}, \tau\right) C_{k}^{T}(\tau) C_{k}(\tau) \Omega_{k}^{T}\left(t_{0}, \tau\right) x_{\alpha} \Delta \tau .
\end{aligned}
$$

Further, we have

$$
\begin{aligned}
\int_{t_{0}}^{t_{f}} y^{T}(\tau) y(\tau) \Delta \tau= & x_{\alpha}^{T} M\left(t_{0}, t_{0}, t_{1}\right) x_{\alpha}+\sum_{i=2}^{k-1}\left[\prod_{j=1}^{i}\left(1+c_{j}\right)\right]^{2} x_{\alpha}^{T} M\left(t_{0}, t_{i-1}, t_{i}\right) x_{\alpha} \\
& +\left[\prod_{j=1}^{k}\left(1+c_{j}\right)\right]^{2} x_{\alpha}^{T} M\left(t_{0}, t_{k-1}, t_{f}\right) x_{\alpha}=0
\end{aligned}
$$

and so,

$$
\int_{t_{0}}^{t_{f}}\|y(\tau)\|^{2} \Delta \tau=0
$$

It follows that

$$
0=y(t)=\left\{\begin{array}{l}
C_{1}(t) \Phi_{A_{1}}^{T}\left(t_{0}, t\right) x_{0}, t \in\left[t_{0}, t_{1}\right]_{\mathbb{T}}, \\
\prod_{j=1}^{l}\left(1+c_{j}\right) C_{l}(t) \Omega_{l}^{T}\left(t_{0}, t\right) x_{0} t \in\left[t_{l-1}, t_{l}\right)_{\mathbb{T}}, \quad l=2, \ldots, k-1, \\
\prod_{j=1}^{k}\left(1+c_{j}\right) C_{k}(t) \Omega_{k}^{T}\left(t_{0}, t\right) x_{0} t \in\left[t_{k-1}, t_{k}\right)_{\mathbb{T}} .
\end{array}\right.
$$


The last equality implies, by Definition 16, that the impulsive system is not observable on $\left[t_{0}, t_{f}\right]_{\mathbb{T}}\left(t_{f} \in\left[t_{k-1}, t_{k}\right) \mathbb{\pi}\right)$.

Our next result give us the sufficient and necessary criterion for time-invarient case for impulsive system (28).

Let us define the following matrix

$$
S=\left[\begin{array}{c}
V_{1} \\
\vdots \\
V_{k}
\end{array}\right] \text { and } V_{i}=\left[\begin{array}{c}
C_{i} \\
C_{i} A_{i}^{T} \\
\vdots \\
C_{i}\left(A_{i}^{T}\right)^{n-1}
\end{array}\right]
$$

Theorem 18. Assume that $1+c_{i} \geq 0, i=1,2, \ldots, k$ and $A_{k}(t)=A_{k}, C_{k}(t)=C_{k}$ are constant matrices. Then, the impulsive system (28) is observable on $\left[t_{0}, t_{f}\right]_{\mathbb{T}}\left(t_{f} \in\left[t_{k-1}, t_{k}\right)_{\mathbb{T}}\right)$ if and only if $\operatorname{rank}(S)=n$.

Proof. Suppose that $\operatorname{rank}(S)=n$ and we aim to show that system (28) is observable on $\left[t_{0}, t_{f}\right]_{\mathbb{T}}\left(t_{f} \in\left[t_{k-1}, t_{k}\right)_{\mathbb{T}}\right)$. If otherwise, namely system (28) is not observable then by Theorem 17, it follows that the matrix $M\left(t_{0}, t_{f}\right)$ is not invertible, which leads to that there exists a nonzero vector $x_{\alpha} \neq 0$. Then by using Theorem 17, we obtain

$$
\begin{aligned}
x_{\alpha}^{T} M\left(t_{0}, t_{0}, t_{1}\right) x_{\alpha} & =\int_{t_{0}}^{t_{1}} x_{\alpha}^{T} e_{A_{1}}\left(t_{0}, \tau\right) C_{1}^{T} C_{1} e_{A_{1}}^{T}\left(t_{0}, \tau\right) x_{\alpha} \Delta \tau \\
& =\int_{t_{0}}^{t_{1}}\left[C_{1} e_{A_{1}}^{T}\left(t_{0}, \tau\right) x_{\alpha}\right]^{T}\left[C_{1} e_{A_{1}}^{T}\left(t_{0}, \tau\right) x_{\alpha}\right] \Delta \tau,
\end{aligned}
$$

it follows that

$$
C_{1} e_{A_{1}}^{T}\left(t_{0}, t\right) x_{\alpha}
$$

Similarly,

$$
C_{i} \Omega_{i}^{T}\left(t_{0}, t\right) x_{\alpha}=0, i=1, \cdots, k-1,
$$

and

$$
C_{k} \Omega_{k}^{T}\left(t_{0}, t\right) x_{\alpha}=0
$$

where

$$
\Omega_{i}^{T}\left(t_{0}, t\right)=e_{A_{i}}^{T}\left(t_{i-1}, t\right) e_{A_{i-1}}^{T}\left(t_{i-2}, t_{i-1}\right) \cdots e_{A_{1}}^{T}\left(t_{0}, t_{1}\right)
$$

Obviously, at $t=t_{0}$, we have $C_{i} x_{\alpha}=0$, for $i=1, \cdots, k$, and differentiating the equations (35), (36) and(37) $n-1$ times and evaluating the result at $t=t_{0}$ gives

$$
C_{i} A_{i}^{j} x_{\alpha}=0, j=0,1, \ldots, n-1, i=1, \cdots, k
$$


Therefore, by (34) and (38) we have $S x_{\alpha}=0$ and moreover $x_{\alpha} \neq 0$ implies that $\operatorname{rank}(S)<n$ which leads to a contradiction with the assumptions that $\operatorname{rank}(S)=n$. The proof of sufficient part is finished.

Conversely, we suppose that $\operatorname{rank}(S)<n$. Then, there exist $v_{\alpha} \neq 0$ such that $S x_{\alpha}=0$, which leads to (38).

By using (14) and (38), we have

$$
\begin{aligned}
M\left(t_{0}, t_{0}, t_{1}\right) x_{\alpha} & =\int_{t_{0}}^{t_{1}} \sum_{j=0}^{n-1} \gamma_{j}\left(t_{0}, \tau\right) e_{A_{1}}\left(t_{0}, \tau\right) C_{1}^{T} C_{1} e_{A_{1}}^{T}\left(t_{0}, \tau\right) x_{\alpha} \Delta \tau \\
& =\int_{t_{0}}^{t_{1}} \sum_{j=0}^{n-1} \gamma_{1 j}\left(t_{0}, \tau\right) e_{A_{1}}\left(t_{0}, \tau\right) C_{1}^{T} C_{1} A_{1}^{T} x_{\alpha} \Delta \tau=0,
\end{aligned}
$$

similarly, for $i=1,2, \ldots, k-1$

$$
M\left(t_{0}, t_{i-1}, t_{i}\right) x_{\alpha}=0
$$

and

$$
M\left(t_{0}, t_{k-1}, t_{k}\right) x_{\alpha}=0
$$

(38) yields $M\left(t_{0}, t_{f}\right) x_{\alpha}=0$. Since $x_{\alpha} \neq 0$, the matrix $M\left(t_{0}, t_{f}\right)$ is not invertible. Hence the system is not observable and it contradicts with the assumption of observability. The proof is completed.

\section{Conclusion}

In this paper, we investigated the issue on the controllability and observability criteria for linear impulsive adjoint system on time scales. Using the adjoint system, we find some necessary and sufficient results of controllability for adjoint impulsive system (for both time varying and time invariant) on time scales. we also find some necessary and sufficient results of observability for adjoint impulsive system (for both time varying and time invariant) on time scales.

\section{References}

[1] M. Adivar, A new periodicity concept for time scales, Math. Slovaca, 63 (2013), no.4, 817-828.

[2] M. Adviar, Principal matrix solutions and variations of parameters for Volterra integro-dynamic equations on time scales, Glasg. Math. J., 53-3 (2011), no.3, 1-18.

[3] R. P. Agarwal, M. Bohner, D. O’Regan and A. Peterson, Dynamic equations on time scales: A survey, J. Comput, Appl. Math., 141 (2002), 1-26.

[4] D. D. Bainov and P. S. Simeonov, Stability Theory of Differential Equations with Impulsive Effect: Theory and Applications. Chichester, U.K. Ellis Horwood, 1989.

[5] M. Bohner and A. Peterson, Advanced in Dynamic Equations on time Scale, Birkhauser, Boston, 2003. 
[6] M. Bohner and A. Peterson, Dynamic Equations on time Scale, An introduction with Applications. Birkhauser, Boston, 2001.

[7] D. Chen, J. Sun and Q. Wu, Impulsive control and its application to Lu's chaotic system, Chaos, Solution, Fractals, 21 (2004), 1135-1142.

[8] J. J. DaCunha, Instsbility results for slowly time varying linear dynamic systems on time scales, J. Math. Anal. Appl., 328 (2007), 1278-1289.

[9] F. Genga, Y. Xu and D. Zhu, Periodic boundary value problems for first order impulsive dynamic equations on time scales, Nonlinear Analysis, 69 (2008), 4074-4087.

[10] Z. H. Guan, T. H. Qian and X. Yu, On controllability and observability for a class of impulsive systems, Systems \& Control Letters, 47 (2002), 247-257.

[11] S. Hilger, Analysis on measure chain-a unified approach to continuous and discrete calculus, Result Math., 18 (1990), 18-56.

[12] J. Hoffacker and C. C. Tisdell, Stability and instability for dynamic equations on time scales, Comput. Math. Appl., 49 (2005), 1327-1334.

[13] B. Kaymakealan, R. Mert and A. Zafer, Asymptotic equivalance of dynamic system on time scale, Discrete and continuous dynamical systems Supplement, (2007), 558-567.

[14] S. Leela, F. A. McRae and S. Sivasundaram, Controllability of impulsive differential equations, J. Math. Anal. Appl., 177 (1993), 24-30.

[15] Z. G. Li, Y. C. B. Soh and X. H. Xu, Lyapunov stability for a class of hybrid dynamic systems, Automatica, 36 (2000), 297-302. tems, IEEE Trans. Autom. Control, 46 (2001), no. 6, 894-897.

[16] Z. G. Li, C. Y. Wen and Y. C. Soh, Analysis and design of impulsive control systems, IEEE Trans. Autom. Control, 46 (2001), no. 6, 894-897.

[17] H. Liu and X. Xiang, A class of the first order impulsive dynamic equation on time scales, Nonlinear Analysis, 69 (2008), 2803-2811.

[18] V. Lupulescu and A. Zada, Linear impulsive dynamical systems on time scales, Electronic J. Qual. theory Differ. Equ., 11 (2010), 1-30.

[19] V. Lupulescu and A. Younus, On controllability and observability for a class of linear impusive dynamic system on time scales, Math. Comput. Modelling, 54 (2011), 1300-1310.

[20] V. Lupulescu and A. Younus, controllability and observability for a class of time-varying impusive system on time scales, Electron. J. Qual. Theory Differ. Equ., 95 (2011), 1-30.

[21] S. Sivasundaram, Stability of dynamic systems on time scales. Nonlinear Dyn. Syst. Theory, 2 (2002), 185-202.

[22] A. Slavik, Product integration on time Scales, Dynam. System Appl., 19 (2010), 97-112.

[23] A. Slavik, Dynamic equation on time scales and generalized ordinary differential equations, J. Math. Anal. Appl., 385 (2012), 534-550.

[24] G. M. Xie and L. Wang, Controllability and observability of a class of linear impulsive systems, J. Math. Anal. Appl., 304 (2005), 336-355.

[25] T. Yang, Impulsive Control Theory, Springer-Verlag Berlin Heidelberg, 2001.

[26] H. Ye, A. N. Michel and L. Hou, Stability theory for hybrid dynamical systems, IEEE Trans. Autom. Control, 43 (1998), no. 4, 461-474.

Centre for Advanced Studies in Pure and Applied Mathematics, Bahauddin Zakariya University, Multan, Pakistan. E-mail: nusyasmin@yahoo.com

Centre for Advanced Studies in Pure and Applied Mathematics, Bahauddin Zakariya University, Multan, Pakistan. E-mail: safiamath@gmail.com

Centre for Advanced Studies in Pure and Applied Mathematics, Bahauddin Zakariya University, Multan, Pakistan.

E-mail: awaissms@yahoo.com

Department of Humanities and Social Sciences, Bahria University, Karachi Campus, Karachi, Pakistan.

E-mail: drasifmansure.bukc@bahria.edu.pk 